\title{
ON THE GENERIC STRUCTURE OF COHOMOLOGY MODULES FOR SEMISIMPLE ALGEBRAIC GROUPS
}

\author{
HENNING HAAHR ANDERSEN ${ }^{1}$
}

\begin{abstract}
Let $G$ be a connected semisimple algebraic group over a field of positive characteristic. Denote by $B$ a Borel subgroup. Our main result says that generically the cohomology modules for line bundles on $G / B$ have simple socles and simple heads, and we identify the corresponding highest weights. As one of the consequences we discover a certain symmetry among extensions of simple modules for $G$.
\end{abstract}

In this paper we continue the study of the module structure of the cohomology groups $H^{*}(\lambda)=H^{*}(G / B, \mathfrak{L}(\lambda))$ for $G$ a semisimple algebraic group over a field of positive characteristic $p, B$ a Borel subgroup of $G$ and $\mathfrak{L}(\lambda)$ the line bundle on $G / B$ induced by the character $\lambda$ of $B$. For earlier work on various aspects of this question see e.g. [1]-[4], [7], [9], [11]-[13].

Here we will consider the case where $\lambda$ is generic, i.e. lies far away from the walls of the Weyl chamber containing it (see $\$ 2.1$ for the precise condition). In this case $H^{*}(\lambda)$ is nonzero in exactly one degree. Our main result, Theorem 2.2 , says that this nonzero cohomology module has simple socle and simple head. For $\lambda$ dominant it is a classical result that $H^{0}(\lambda)$ has simple socle. The first nondominant case was treated in [2] where we proved that $H^{1}(\lambda)$ also always has a simple socle. It should likewise be mentioned that J. C. Jantzen has proved that Weyl modules (which may be identified with $H^{N}(\lambda), N=\operatorname{dim} G / B$ ) in the generic case have simple socles [12, 6.3] and that J. E. Humphreys in [10] has formulated conjectures which suggest that for $\lambda$ generic, $H^{*}(\lambda)$ has the same module structure as a Weyl module. Recently, Doty and Sullivan have announced results which include special cases of our result (see their forthcoming paper [8]).

To formulate some of the consequences of our main theorem, we need a little more notation. We set $W$ equal to the Weyl group of $G$ relative to a maximal torus $T$ in $B$. For $w \in W$ we denote by $l(w)$ the length of $w$ and if $\lambda$ is a generic dominant weight, then we let $L\left(\lambda^{w}\right)$ be the simple $G$-module which by our theorem occurs as the socle of $H^{l(w)}(w \cdot \lambda)$.

It follows easily (see §2.4) from our proof that for any $y, w \in W$ the simple module $L\left(\lambda^{w}\right)$ occurs exactly once as a composition factor of $H^{l(y)}(y \cdot \lambda)$ and this allows us to prove (see Proposition 2.4) that up to scalar there exists a unique

Received by the editors February 19, 1985.

1980 Mathematics Subject Classification (1985 Revision). Primary 20G05; Secondary 14L15.

${ }^{1}$ Supported in part by the Danish Natural Science Research Council. 
nonzero homomorphism between $H^{l(w)}(w \cdot \lambda)$ and $H^{l(y)}(y \cdot \lambda)$. In the case where $\lambda$ is located in the lowest $p^{2}$-alcove we are moreover able to determine the filtration level of $L\left(\lambda^{w}\right)$ in $H^{l(y)}(y \cdot \lambda)$ with respect to the filtration constructed in [4] (see Proposition 2.7 below).

Another application of our method is to the question of extensions between simple $G$-modules. Here we discover a certain symmetry with respect to the Weyl group (see Theorem 3.6). Finally in the last section we consider the relations between the above homomorphisms $H^{l(w)}(w \cdot \lambda) \rightarrow H^{l(y)}(y \cdot \lambda)$ and homomorphisms between cohomology modules of the same degree but associated to weights in adjacent alcoves.

Our technique is to take advantage of the infinitesimal subgroup schemes $G_{r}$ of $G$ (the kernels of the powers of the Frobenius homomorphism on $G$ ). We study induction from $B$ to $G$ and its derived functors by first inducing from $B$ to the subgroup scheme $B G_{r}$ and then to $G$. This technique was used by E. Cline, B. Parshall and L. Scott in [7] and in particular the results in the appendix of [7] are closely related to some of our results here.

I should like to thank E. Cline and J. E. Humphreys for valuable discussions on the topics treated in this paper. I also gratefully acknowledge the hospitality of the Institute for Advanced Study, Princeton, where the work on this paper was done.

1. Preliminaries. In this section we start by fixing the notation and we then recall some properties of induced modules. We also give some estimates on the weights of the $G_{n} B$-modules induced from characters of $B$ which we shall need later on. Finally, we define the weights $\lambda^{w}$ mentioned in the introduction and examine some of their properties.

1.1. The following notation will be used throughout the paper.

By $k$ we denote an algebraically closed field of characteristic $p>0$. We assume that the connected semisimple algebraic group $G$ over $k$ is simply connected. The character group of the maximal torus $T$ in $B$ is denoted $X(T)$ and in the root system $R \subset X(T)$ associated to $(G, T)$ we choose, as usual, the set of positive roots $R_{+}$such that $B$ corresponds to $R_{-}=-R_{+}$. We let $S$ denote the set of simple roots and the Weyl group $W$ is then generated by the reflections $s_{\alpha}, \alpha \in S$. (Alternatively $W=N_{G}(T) / T$.) On $W$ we have the length function $l$ and we let $w_{0}$ denote the element of maximal length.

We set $\rho$ equal to half the sum of the positive roots or equivalently $\rho=\sum_{\beta \in S} \omega_{\beta}$, where $\omega_{\beta}$ denotes the fundamental weight associated to $\beta \in S$. Then the "dot" action of $W$ on $X(T)$ is given by $w \cdot \lambda=w(\lambda+\rho)-\rho, \lambda \in X(T), w \in W$.

If $R$ is indecomposable we let $\alpha_{0}$ denote the highest short root and we set $h=\left\langle\alpha_{0}^{v}, \rho\right\rangle+1$ (the Coxeter number). In general we let $h$ denote the maximum of the Coxeter numbers of the indecomposable components of $R$.

Fix $n>0$ and set $q=p^{n}$. The set of $q$-restricted weights is

$$
X_{n}(T)=\left\{\lambda \in X(T) \mid 0 \leqslant\left\langle\alpha^{v}, \lambda\right\rangle<q \text { for all } \alpha \in S\right\} .
$$

Then $X_{n}(T)$ is a subset of the set of dominant weights

$$
X(T)_{+}=\left\{\lambda \in X(T) \mid\left\langle\alpha^{v}, \lambda\right\rangle \geqslant 0 \text { for all } \alpha \in S\right\} .
$$


We also define the lowest $q$-alcove and its closure

$$
C_{n}=\left\{\lambda \in X(T)_{+} \mid\left\langle\alpha^{v}, \lambda+\rho\right\rangle<q \text { for all } \alpha \in R_{+}\right\}
$$

respectively

$$
\overline{C_{n}}=\left\{\lambda \in X(T) \mid 0 \leqslant\left\langle\alpha^{v}, \lambda+\rho\right\rangle \leqslant q \text { for all } \alpha \in R_{+}\right\} .
$$

Any element $\lambda \in X(T)$ has a unique decomposition of the form $\lambda=\lambda^{0}+q \lambda^{1}$ with $\lambda^{0} \in X_{n}(T)$ and $\lambda^{1} \in X(T)$. Throughout this paper upper indices 0 and 1 on a weight always refer to this decomposition.

When $V$ is a $T$-module and $\lambda \in X(T)$ we let $V_{\lambda}$ denote the $\lambda$-weight space of $T$ in $V$. We write

$$
\operatorname{ch} V=\sum_{\lambda \in X(T)} \operatorname{dim} V_{\lambda} e^{\lambda} \in \mathbf{Z}[X(T)]
$$

for the formal character of $V$.

If $V$ is a module for an algebraic group scheme $H$ and $L$ is a simple $H$-module we denote by $[V: L]$ the composition factor multiplicity of $L$ in $V$. This notion extends to virtual representations. In the case where $H=G$ this means to $\mathbf{Z}[X(T)]^{W}$.

Let $F: G \rightarrow G$ denote the Frobenius homomorphism. We set $G_{1}$ equal to the (scheme-theoretic) kernel of $F$ and, more generally, $G_{n}$ equal to the kernel of $F^{n}$. Similarly we have the subgroup schemes $T_{n}, B_{n}, \ldots$ of $T, B, \ldots$

If $V$ is a $G$-module given by the homomorphism $\eta: G \rightarrow \mathrm{GL}(V)$, then we denote by $V^{(n)}$ the $G$-module given by $\eta \circ F^{n}$. Note that $V$ equals $V_{1}^{(n)}$ for some $G$-module $V_{1}$ if and only if $\eta\left(G_{n}\right)=1$, i.e. $G_{n}$ operates trivially on $V$. Again similar notation is used for $T, B, \ldots$-modules.

1.2. When $H$ is a closed subgroup of $G$ and $E$ is an $H$-module we have the induced $G$-module $\operatorname{Ind}_{H}^{G} E$ which together with the natural $H$-homomorphism $E v$ : $\operatorname{Ind}_{H}^{G} E \rightarrow E$ satisfy Frobenius reciprocity

$$
\operatorname{Hom}_{G}\left(V, \operatorname{Ind}_{H}^{G} E\right) \stackrel{\sim}{\rightarrow} \operatorname{Hom}_{H}(V, E)
$$

for all $G$-modules $V$. Here the map takes $f \in \operatorname{Hom}_{G}\left(V, \operatorname{Ind}_{H}^{G} E\right)$ into $E v \circ f$.

We also have the so-called tensor identity

$$
\operatorname{Ind}_{H}^{G}(V \otimes E) \simeq V \otimes \operatorname{Ind}_{H}^{G} E .
$$

The functor $\operatorname{Ind}_{H}^{G}$ from rational $H$-modules to rational $G$-modules has right derived functors $H^{i}(G / H,-), i \geqslant 0$. When $H=B$ we write $H^{i}(-)=H^{i}(G / B,-)$.

Of particular importance are the $G$-modules $H^{i}(\lambda)$, where $\lambda \in X(T)$ is considered a $B$-module via the natural homomorphism $B \rightarrow T$. Recall that

$$
H^{0}(\lambda) \neq 0 \text { if and only if } \lambda \in X(T)_{+} .
$$

For $\lambda \in X(T)_{+}, H^{0}(\lambda)$ contains a unique simple submodule $L(\lambda)$ and in this way $X(T)_{+}$parametrizes all simple $G$ modules. 
1.3. The concept of induction in $\S 1.2$ generalizes to subgroup schemes with all formal properties (e.g. (1) and (2) in \$1.2) preserved. In particular we shall consider induction from $B_{n}$ to $G_{n}$, respectively $B$ to $G_{n} B$. Here we use the notation

$$
Z_{n}(E)=\operatorname{Ind}_{B_{n}}^{G_{n}} E, \quad \text { respectively } \hat{Z}_{n}(E)=\operatorname{Ind}_{B}^{G_{n} B} E,
$$

where $E$ is a $B_{n}$-module, respectively a $B$-module. When $E$ is a $B$-module we have $Z_{n}(E)=\left.\hat{Z}_{n}(E)\right|_{G_{n}}$.

Note that since $G_{n} / B_{n} \simeq G_{n} B / B$ is affine, both functors $Z_{n}$ and $\hat{Z}_{n}$ are exact so that we do not have any derived functors.

Again the modules $\hat{Z}_{n}(\lambda), \lambda \in X(T)$, are of particular importance. We shall need the following properties (see [7 or 12]), valid for all $\lambda \in X(T)$

$$
\hat{Z}_{n}(\lambda) \simeq \hat{Z}_{n}\left(\lambda^{0}\right) \otimes q \lambda^{1} .
$$

The $G_{n} B$-module $\hat{Z}_{n}(\lambda)$ contains a unique simple submodule $\hat{L}_{n}(\lambda)$ and in this way $X(T)$ parametrizes the isomorphism classes of simple $G_{n} B$-modules.

$$
\begin{gathered}
\hat{L}_{n}(\lambda) \simeq L\left(\lambda^{0}\right) \otimes q \lambda^{1} . \\
\hat{Z}_{n}((q-1) \rho)=\hat{L}_{n}((q-1) \rho)=L((q-1) \rho) .
\end{gathered}
$$

This module is called the $n$th Steinberg module and alternatively denoted $\mathrm{St}_{n}$.

$$
\begin{gathered}
\operatorname{ch} \hat{Z}_{n}(\lambda)=\operatorname{ch~St}_{n} e^{\lambda-(q-1) \rho} . \\
\hat{Z}_{n}(\lambda)^{*} \simeq \hat{Z}_{n}(2(q-1) \rho-\lambda) .
\end{gathered}
$$

1.4. In analogy with Jantzen, e.g. $[12,5.1]$, we say that a filtration of a $G_{n} B$ module $V$,

$$
0=V_{0} \subset V_{1} \subset \cdots \subset V_{r}=V,
$$

is a $\hat{Z}_{n}$-filtration if there exists $\lambda_{i} \in X(T)$ such that $V_{i} / V_{i-1} \simeq \hat{Z}_{n}\left(\lambda_{i}\right), i=1, \ldots, r$.

When $V$ possesses such a filtration we set

$$
\left[V: \hat{Z}_{n}(\lambda)\right]=\#\left\{i \mid V_{i} / V_{i-1} \simeq \hat{Z}_{n}(\lambda)\right\}
$$

for all $\lambda \in X(T)$. The exactness of $\hat{Z}_{n}$ and the tensor identity (the analogue of 1.2(2)) show immediately

If $V$ has a $\hat{Z}_{n}$-filtration so has $V \otimes V^{\prime}$ for any $G_{n} B$-module

$$
V^{\prime} \text { and }\left[V \otimes V^{\prime}: \hat{Z}_{n}(\lambda)\right]=\sum_{\nu \in X(T)}\left[V: \hat{Z}_{n}(\nu)\right] \operatorname{dim} V_{\lambda-\nu}^{\prime} \text {. }
$$

1.5. Let $\lambda \in X_{n}(T)$. In the following we shall assume that there exists a $G$ summand $Q_{n}(\lambda)$ of $\mathrm{St}_{n} \otimes L\left((q-1) \rho+w_{0}(\lambda)\right)$ such that $L(\lambda)$ is the only simple $G_{n}$-submodule of $Q_{n}(\lambda)$. This is known to hold for $p \geqslant 2(h-1)$ (see [12, Corollary 4.5]).

For general $\lambda \in X(T)$ we set

$$
\hat{Q}_{n}(\lambda)=Q_{n}\left(\lambda^{0}\right) \otimes q \lambda^{1} .
$$


Then $\hat{Q}_{n}(\lambda)$ is a $G_{n} B$-module which contains $\hat{L}_{n}(\lambda)$ as its only simple $G_{n} B$-submodule. Being a direct summand of $\mathrm{St}_{n} \otimes L\left((q-1) \rho+w_{0}\left(\lambda^{0}\right)\right) \otimes q \lambda^{1}$ it follows from 1.4(1) that

$$
\hat{Q}_{n}(\lambda) \text { has a } \hat{Z}_{n} \text {-filtration. }
$$

Since $\mathrm{St}_{n}$ is injective as a $G_{n} T$-module so is $\hat{Q}_{n}(\lambda)$ and it is then easy to derive the following reciprocity law

$$
\left[\hat{Q}_{n}(\lambda): \hat{Z}_{n}(\mu)\right]=\left[\hat{Z}_{n}(\mu): \hat{L}_{n}(\lambda)\right] \text { for all } \mu \text {. }
$$

1.6. As Ind $G_{G_{n} B}^{G} \circ \hat{Z}_{n}=\operatorname{Ind}_{B}^{G}$ and as $\hat{Z}_{n}$ is exact and takes injective $B$-modules into injective $G_{n} B$-modules we get

$$
H^{i}(E) \simeq H^{i}\left(G / G_{n} B, \hat{Z}_{n}(E)\right)
$$

for all $B$-modules $E$.

If $E$ is a $B$-module, then $E^{(n)}$ is a $G_{n} B$-module (via the composite $G_{n} B \stackrel{F}{\rightarrow} B \rightarrow$ $\mathrm{GL}(E))$. As $G_{n}$ acts trivially on $E^{(n)}$ it will also act trivially on $\operatorname{Ind}_{G_{n} B}^{G}\left(E^{(n)}\right)$, i.e. there exists a $G$-module $V$ such that $\operatorname{Ind}_{G_{n} B}^{G}\left(E^{(n)}\right) \simeq V^{(n)}$. We claim that $V \simeq \operatorname{Ind}_{B}^{G} E$. To see this it is enough to prove that $V$ has the right universal property. So let $V_{1}$ be another $G$-module. Then we have

$$
\begin{aligned}
\operatorname{Hom}_{G}\left(V_{1}, V\right) & \simeq \operatorname{Hom}_{G / G_{n}}\left(V_{1}^{(n)}, V^{(n)}\right) \simeq \operatorname{Hom}_{G / G_{n}}\left(V_{1}^{(n)}, \operatorname{Ind}_{G_{n} B}^{G}\left(E^{(n)}\right)\right) \\
& \simeq \operatorname{Hom}_{B / B_{n}}\left(V_{1}^{(n)}, E^{(n)}\right) \simeq \operatorname{Hom}_{B}\left(V_{1}, E\right) .
\end{aligned}
$$

It follows easily that we have the same relation between the derived functors, i.e.

$$
H^{i}\left(G / G_{n} B, E^{(n)}\right) \simeq H^{i}(E)^{(n)}
$$

for all $i \geqslant 0$ and all $B$-modules $E$. This result was proved by Cline, Parshall and Scott in a more general context (see [6, 4.3]).

1.7. LemMA. Let $\lambda \in X(T)$. The weights $\nu$ of $\hat{Z}_{n}(\lambda)$ satisfy the inequalities

$$
\left|\left\langle\alpha^{v}, \lambda^{1}-\left(\nu^{1}+\rho\right)\right\rangle\right|<2(h-1) \quad \text { for all } \alpha \in R \text {. }
$$

Proof. Assume that $R$ is indecomposable and let $\alpha_{0}$ denote the largest short root. By 1.3(5) we see that $\nu$ is a weight of $\hat{Z}_{n}(\lambda)$ if and only if $\nu-\lambda+(q-1) \rho$ is a weight of $\mathrm{St}_{n}$. Now any weight $\mu$ of $\mathrm{St}_{n}$ satisfies

$$
\begin{aligned}
\max _{\alpha \in R}\left\{\left|\left\langle\alpha^{v}, \mu\right\rangle\right|\right\} & =\max _{\alpha \in R}\left\{\left|\left\langle\alpha^{v}, w(\mu)\right\rangle\right|\right\} \\
& =\left\langle\alpha_{0}^{v}, w(\mu)\right\rangle \leqslant\left\langle\alpha_{0}^{v},(q-1) \rho\right\rangle=(q-1)(h-1) .
\end{aligned}
$$

Here $w$ is the element of $W$ for which $w(\mu) \in X(T)_{+}$. Using this we find

$$
\begin{aligned}
\left|\left\langle\boldsymbol{\alpha}^{v}, \nu-\lambda+(q-1) \rho\right\rangle\right| & =\left|\left\langle\boldsymbol{\alpha}^{v}, \nu^{0}-\lambda^{0}\right\rangle+q\left\langle\boldsymbol{\alpha}^{v}, \nu^{1}+\rho-\lambda^{1}\right\rangle-\left\langle\boldsymbol{\alpha}^{v}, \rho\right\rangle\right| \\
& <(q-1)(h-1) .
\end{aligned}
$$

As $\nu^{0}, \lambda^{0} \in X_{n}(T)$ we have $\left|\left\langle\alpha^{v}, \nu^{0}-\lambda^{0}\right\rangle\right| \leqslant\left\langle\alpha_{0}^{v},(q-1) \rho\right\rangle=(q-1)(h-1)$. Also $\left\langle\boldsymbol{\alpha}^{v}, \rho\right\rangle \mid \leqslant h-1$ so the above inequality gives

$$
q\left|\left\langle\alpha^{v}, \nu^{1}+\rho-\lambda^{1}\right\rangle\right| \leqslant 2(q-1)(h-1)+h-1<2 q(h-1) .
$$


1.8. Let $\lambda \in X_{n}(T)$. For each $w \in W$ we let $\lambda_{w} \in X(T)$ be determined by the requirement $q \lambda_{w}+w \cdot \lambda \in X_{n}(T)$. This means that if we let $n_{\alpha}^{\lambda}$ be given by $n_{\alpha}^{\lambda} q\left\langle\left\langle\alpha^{v}, \lambda+\rho\right\rangle \leqslant\left(n_{\alpha}^{\lambda}+1\right) q\right.$ for $\alpha \in R$, then

$$
\lambda_{w}=-\sum_{\beta \in S} n_{w^{-1}(\beta)}^{\lambda} \omega_{\beta} .
$$

In fact, if $\lambda_{w}$ is defined by (1), then we get that $\left\langle\beta^{v}, q \lambda_{w}+w \cdot \lambda\right\rangle=-q n_{w^{-1}(\beta)}^{\lambda}+$ $\left\langle w^{-1}(\beta)^{v}, \lambda+\rho\right\rangle-1$ lies between 0 and $q-1$ for each $\beta \in S$.

If $\lambda \in X(T)$ we set $\lambda^{w}=q \lambda_{w}^{0}+w \cdot \lambda^{0}+q\left(\lambda^{1}+w^{-1} \cdot\left(-\lambda_{w}^{0}\right)\right)$. Note that we can write this also as $\lambda^{w}=(w \cdot \lambda)^{0}+q w^{-1} \cdot\left((w \cdot \lambda)^{1}\right)$. We shall need the following properties of $\lambda^{w}$.

LEMMA. With the above notation we have for all $\lambda \in X(T)$ and all $w \in W$

(i) $\left|\left\langle\alpha^{v}, \lambda_{w}^{0}\right\rangle\right| \leqslant 2(h-1)$ for all $\alpha \in R$.

(ii) $\left|\left\langle\alpha^{v}, \rho-\lambda_{w}^{0}\right\rangle\right| \leqslant 2(h-1)$ for all $\alpha \in R$.

(iii) $-w_{0}\left(\left(-w_{0}\left(\lambda^{0}\right)\right)_{w}\right)=\lambda_{w_{0} w w_{0}}^{0}$

(iv) $-w_{0}\left(\left(w_{0}(\lambda)\right)^{w}\right)=\lambda^{w_{0} w w_{0}}$.

Proof. We assume that $R$ is indecomposable. To prove (i) note that for any $\alpha \in R$ we have

$$
\left|\left\langle\boldsymbol{\alpha}^{v}, q \lambda_{w}^{0}+w\left(\lambda^{0}+\rho\right)\right\rangle\right| \leqslant\left\langle\boldsymbol{\alpha}_{0}^{v}, q \lambda_{w}^{0}+w\left(\lambda^{0}+\rho\right)\right\rangle \leqslant\left\langle\boldsymbol{\alpha}_{0}^{v}, q \rho\right\rangle=q(h-1)
$$

and hence

$$
\begin{aligned}
q\left|\left\langle\boldsymbol{\alpha}^{v}, \lambda_{w}^{0}\right\rangle\right| & \leqslant q(h-1)+\left|\left\langle\boldsymbol{\alpha}^{v}, w\left(\lambda^{0}+\rho\right)\right\rangle\right| \\
& \leqslant q(h-1)+\left\langle\boldsymbol{\alpha}_{0}^{v}, \lambda^{0}+\rho\right\rangle \leqslant 2 q(h-1) .
\end{aligned}
$$

A completely analogous argument proves (ii). Now for (iii) we have to show that $-q w_{0}\left(\left(-w_{0}\left(\lambda^{0}\right)\right)_{w}\right)+w_{0} w w_{0} \cdot \lambda \in X_{n}(T)$. As $-w_{0}$ maps $X_{n}(T)$ into itself this is equivalent to showing that $q\left(-w_{0}\left(\lambda^{0}\right)\right)_{w}-w_{0}\left(w_{0} w w_{0} \cdot \lambda\right) \in X_{n}(T)$. But

$$
-w_{0}\left(w_{0} w w_{0} \cdot \lambda\right)=-w w_{0}\left(\lambda^{0}+\rho\right)-\rho=w \cdot\left(-w_{0}\left(\lambda^{0}\right)\right)
$$

and by definition of $-w_{0}\left(\lambda^{0}\right)_{w}$ we have $q\left(-w_{0}\left(\lambda^{0}\right)\right)_{w}+w \cdot\left(-w_{0}\left(\lambda^{0}\right)\right) \in X_{n}(T)$. Finally this also easily gives (iv) because by (iii) we have

$$
\begin{aligned}
\lambda^{w_{0} w w_{0}} & =-q w_{0}\left(\left(-w_{0}\left(\lambda^{0}\right)\right)_{w}\right)+w_{0} w w_{0} \cdot \lambda^{0}+q\left(\lambda^{1}+w_{0} w^{-1} w_{0} \cdot\left(w_{0}\left(\left(-w_{0}\left(\lambda^{0}\right)\right)_{w}\right)\right)\right) \\
& =-w_{0}\left(q\left(-w_{0}\left(\lambda^{0}\right)\right)_{w}\right)+w \cdot\left(-w_{0}\left(\lambda^{0}\right)\right)+q\left(-w_{0}\left(\lambda^{1}\right)+w^{-1} \cdot\left(-\left(-w_{0}\left(\lambda^{0}\right)\right)_{w}\right)\right) .
\end{aligned}
$$

2. On the generic structure of cohomology modules. In this section we shall show that for certain dominant weights $\lambda$ the cohomology modules $H^{l(w)}(w \cdot \lambda), w \in W$, all have simple $G$-socles. In fact, we determine the $G_{n}$-socles of these modules. We also show that for the same values of $\lambda$ we have $\operatorname{Hom}_{G}\left(H^{l(y)}(y \cdot \lambda), H^{l(w)}(w \cdot \lambda)\right)$ $=k$ for all $y, w \in W$ and that the socle of $H^{l(w)}(w \cdot \lambda)$ is realized as the image of the homomorphism $H^{l\left(w_{0} w\right)}\left(w_{0} w \cdot \lambda\right) \rightarrow H^{l(w)}(w \cdot \lambda)$. This allows us to determine the filtration level (with respect to the filtration defined in [4]) of the corresponding composition factor of $H^{(y)}(y \cdot \lambda)$.

2.1. Let $\lambda \in X(T)_{+}$. We call $\lambda$ generic if the following condition is satisfied

$$
6(h-1) \leqslant\left\langle\alpha^{v}, \lambda^{1}\right\rangle \leqslant p-6(h-1) \quad \text { for all } \alpha \in R_{+} .
$$


Note that generic weights exist only for $p>12(h-1)$.

It should be pointed out that this concept is not exactly the same as the corresponding one used in [3, p. 11] nor the one used in the appendix of [7]. The following proposition which is a reformulation of Proposition A.1(b) in [7] shows, however, that generic in the above sense implies generic in the sense of both [3] and [7].

Proposition (Cline, Parshall, Scott [7]). Let $\lambda \in X(T)_{+}$be generic. Then for each $w \in W$ we have

$$
H^{i}(w \cdot \lambda)=0 \quad \text { for } i \neq l(w)
$$

and

$$
\left[H^{l(w)}(w \cdot \lambda): L(\nu)\right]=\left[\hat{Z}_{n}(w \cdot \lambda): \hat{L}_{n}\left(\nu^{0}+q w \cdot \nu^{1}\right)\right]
$$

for all $\nu \in X(T)_{+}$.

Proof. Let $w \in W$ and suppose $i \neq l(w)$. By 1.6(1) we have $H^{i}(w \cdot \lambda) \simeq$ $H^{i}\left(G / G_{n} B, \hat{Z}_{n}(w \cdot \lambda)\right)$. Hence clearly $H^{i}(w \cdot \lambda)=0$ if $H^{i}\left(G / G_{n} B, \hat{L}_{n}(\nu)\right)=0$ for all $\nu \in X(T)$ with $\left[\hat{Z}_{n}(w \cdot \lambda): \hat{L}_{n}(\nu)\right] \neq 0$. Now by $1.6(2)$ and the tensor identity we have $H^{i}\left(G / G_{n} B, \hat{L}_{n}(\nu)\right) \simeq L\left(\nu^{0}\right) \otimes H^{i}\left(\nu^{1}\right)^{(n)}$ and so we have to prove that $H^{i}\left(\nu^{1}\right)$ $=0$ for all $\nu$ as above. This will follow if we can show that $\nu^{1} \in w \cdot \bar{C}_{1}$. But $\nu$ is certainly a weight of $\hat{Z}_{n}(w \cdot \lambda)$ and so by Lemma 1.7 we have

$$
\left|\left\langle\alpha^{v},(w \cdot \lambda)^{1}-\left(\nu^{1}+\rho\right)\right\rangle\right|<2(h-1) \text { for all } \alpha \in R \text {. }
$$

As $(w \cdot \lambda)^{1}=w\left(\lambda^{1}\right)-\lambda_{w}^{0}$ we get for all $\alpha \in R$

$$
\begin{aligned}
\left|\left\langle\alpha^{v}, w^{-1}\left(\nu^{1}+\rho\right)\right\rangle\right| & =\left|\left\langle w(\alpha)^{v}, \nu^{1}+\rho\right\rangle\right|\left\langle 2(h-1)-\left|\left\langle w(\alpha)^{v}, w\left(\lambda^{1}\right)-\lambda_{w}^{0}\right\rangle\right|\right. \\
& \leqslant 2(h-1)+\left|\left\langle\alpha^{v}, \lambda^{1}\right\rangle\right|+\left|\left\langle w(\alpha)^{v}, \lambda_{w}^{0}\right\rangle\right|<p .
\end{aligned}
$$

Here the last inequality uses that $\lambda$ is generic and that $\left|\left\langle w(\alpha)^{v}, \lambda_{w}^{0}\right\rangle\right| \leqslant 2(h-1)$ by Lemma 1.8(i). Similarly, we get $\left\langle\alpha^{v}, w^{-1}\left(\nu^{1}+\rho\right)\right\rangle>0$ for all $\alpha \in R_{+}$by using that $\left\langle\alpha^{v}, \lambda^{1}\right\rangle>4(h-1)$. Thus we have proved $\nu^{1} \in w \cdot C_{1}$ and this concludes the proof of the first statement in this proposition.

The above reasoning also shows that if we apply $H^{l(w)}\left(G / G_{n} B,-\right)$ to a composition series for $\hat{Z}_{n}(w \cdot \lambda)$, then we get a filtration of $H^{l(w)}(w \cdot \lambda)$ whose factors are $H^{l(w)}\left(G / G_{n} B, \hat{L}_{n}(\nu)\right) \simeq L\left(\nu^{0}\right) \otimes H^{l(w)}\left(\nu^{1}\right)^{(n)}$. For all $\nu$ in question the above shows that $\nu^{1} \in w \cdot C_{1}$ and hence $H^{l(w)}\left(\nu^{1}\right) \simeq H^{0}\left(w^{-1} \cdot \nu^{1}\right) \simeq L\left(w^{-1} \cdot \nu^{1}\right)$. Thus $H^{l(w)}\left(G / G_{n} B,-\right)$ takes a $G_{n} B$-composition series for $\hat{Z}_{n}(w \cdot \lambda)$ into a $G$-composition series for $H^{l(w)}(w \cdot \lambda)$.

REMARK. The proof shows that for this proposition we could replace 6 by 4 in condition (1). However, some of the following results require a 6 and we have chosen to stick to this uniform condition throughout.

2.2. When $M$ is a module for an algebraic group scheme $H$ we denote by $\operatorname{Soc}_{H} M$ the $H$-socle of $M$, i.e. Soc $_{H} M$ is the direct sum of all the simple $H$-submodules of $M$. By $\mathrm{Hd}_{H} M$ we denote the head of $M$, i.e. the direct sum of all the simple $H$-quotients of $M$. 
From the proof of Proposition 2.1 we see that for $\lambda$ generic $L\left(\lambda^{w}\right) \simeq$ $H^{l(w)}\left(G / G_{n} B, \hat{L}_{n}(w \cdot \lambda)\right)$ is contained in $\operatorname{Soc}_{G} H^{l(w)}(w \cdot \lambda)$ for all $w \in W$. We shall now prove that in fact equality holds.

TheOREM. Let $\lambda \in X(T)_{+}$be generic. Then for all $w \in W$ we have

$$
\operatorname{Soc}_{G_{n}} H^{l(w)}(w \cdot \lambda)=L\left(\lambda^{w}\right)=\operatorname{Soc}_{G} H^{l(w)}(w \cdot \lambda)
$$

and

$$
\operatorname{Hd}_{G_{n}} H^{l(w)}(w \cdot \lambda)=L\left(\lambda^{w_{0} w}\right)=\operatorname{Hd}_{G} H^{l(w)}(w \cdot \lambda) .
$$

Proof. Let $w \in W$. From $\S 1.5$ it follows immediately that $\hat{Z}_{n}(w \cdot \lambda)$ is a $G_{n} B$-submodule of $\hat{Q}_{n}(w \cdot \lambda)$ and that the quotient $\hat{Q}_{n}(w \cdot \lambda) / \hat{Z}_{n}(w \cdot \lambda)$ has a $\hat{Z}_{n}$-filtration with $\hat{Z}_{n}(\mu)$ occurring $\left[\hat{Z}_{n}(\mu): \hat{L}_{n}(w \cdot \lambda)\right]$ times, $\mu \neq w \cdot \lambda$. Suppose now $\left[\hat{Z}_{n}(\mu): \hat{L}_{n}(w \cdot \lambda)\right] \neq 0$ and let $\hat{L}_{n}(\nu)$ be a composition factor of $\hat{Z}_{n}(\mu)$. We shall show that $\nu^{1} \in w \cdot \bar{C}_{1}$. Then $H^{i}(\mu)=H^{i}\left(G / G_{n} B, \hat{Z}_{n}(\mu)\right)=0$ for $i \neq l(w)$ by the arguments in the proof of Proposition 2.1.

Using Lemma 1.7 we get

$$
\begin{aligned}
\left\langle\alpha^{v}, \nu^{1}+\rho\right\rangle & =\left\langle\alpha^{v}, \nu^{1}+\rho-\mu^{1}\right\rangle+\left\langle\alpha^{v}, \mu^{1}-(w \cdot \lambda)^{1}-\rho\right\rangle+\left\langle\alpha^{v},(w \cdot \lambda)^{1}+\rho\right\rangle \\
& \leqslant 4(h-1)+\left\langle\alpha^{v},(w \cdot \lambda)^{1}+\rho\right\rangle
\end{aligned}
$$

and similarly the same expression has absolute value

$$
\geqslant\left|\left\langle\alpha^{v},(w \cdot \lambda)^{1}+\rho\right\rangle\right|-4(h-1) .
$$

Now $(w \cdot \lambda)^{1}=w\left(\lambda^{1}\right)-\lambda_{w}^{0}$ and as $\lambda$ is generic, we have that $\left\langle\alpha^{v},(w \cdot \lambda)^{1}+\rho\right\rangle=$ $\left\langle w^{-1}(\alpha), \lambda^{1}\right\rangle+\left\langle\alpha^{v}, \rho-\lambda_{w}^{0}\right\rangle$ lies between $4(h-1)$ and $p-4(h-1)$ (see Lemma 1.8(ii)) for all $\alpha$ with $w^{-1}(\alpha) \in R_{+}$. Combined with the above, this shows $\nu^{1} \in w \cdot C_{1}$.

In particular we get from this that $H^{l(w)-1}\left(G / G_{n} B, \hat{Q}_{n}(w \cdot \lambda) / \hat{Z}_{n}(w \cdot \lambda)\right)=0$ so that we have an inclusion

$$
H^{l(w)}(w \cdot \lambda) \simeq H^{l(w)}\left(G / G_{n} B, \hat{Z}_{n}(w \cdot \lambda)\right) \subset H^{l(w)}\left(G / G_{n} B, \hat{Q}_{n}(w \cdot \lambda)\right) .
$$

Using the fact $\hat{Q}_{n}(w \cdot \lambda)=Q_{n}\left((w \cdot \lambda)^{0}\right) \otimes q(w \cdot \lambda)^{1}$, the tensor identity and the isomorphism 1.6(2) we find

$$
H^{l(w)}\left(G / G_{n} B, \hat{Q}_{n}(w \cdot \lambda)\right) \simeq Q_{n}\left((w \cdot \lambda)^{0}\right) \otimes H^{l(w)}\left((w \cdot \lambda)^{1}\right)^{(n)} .
$$

As we have seen before

$$
H^{l(w)}\left((w \cdot \lambda)^{1}\right) \simeq H^{0}\left(w^{-1} \cdot\left((w \cdot \lambda)^{1}\right)\right) \simeq L\left(w^{-1} \cdot\left((w \cdot \lambda)^{1}\right)\right)
$$

and since the $G_{n}$-socle of $Q_{n}\left((w \cdot \lambda)^{0}\right)$ is $L\left((w \cdot \lambda)^{0}\right)$ we see

$$
\operatorname{Soc}_{G_{n}} H^{l(w)}\left(G / G_{n} B, \hat{Q}_{n}(w \cdot \lambda)\right)=L\left((w \cdot \lambda)^{0}\right) \otimes L\left(w^{-1} \cdot(w \cdot \lambda)^{1}\right)^{(n)}=L\left(\lambda^{w}\right) .
$$

This proves the first assertion in the theorem. The second then also follows because by Serre duality we have

$$
H^{l(w)}(w \cdot \lambda)^{*} \simeq H^{N-l(w)}(-w \cdot \lambda-2 \rho)=H^{N-l(w)}\left(w w_{0} \cdot\left(-w_{0}(\lambda)\right)\right)
$$


and therefore

$$
\begin{aligned}
\operatorname{Hd}_{G_{n}}\left(H^{l(w)}(w \cdot \lambda)\right) & =\left(\operatorname{Soc}_{G_{n}} H^{l(w)}(w \cdot \lambda)^{*}\right) * \\
& =\left(\operatorname{Soc}_{G_{n}} H^{N-l(w)}\left(w w_{0} \cdot\left(-w_{0}(\lambda)\right)\right)\right)^{*} \\
& =L\left(\left(-w_{0}(\lambda)\right)^{w w_{0}}\right) *=L\left(-w_{0}\left(\left(-w_{0}(\lambda)\right)^{w w_{0}}\right)\right)=L\left(\lambda^{w_{0} w}\right),
\end{aligned}
$$

where the last equality follows from Lemma 1.8(iv).

2.3. The examples we have worked out (for rank 2 groups) indicate that the condition on $\lambda$ in 2.2 is stronger than necessary. However, the vanishing behaviour of $H^{i}(w \cdot \lambda)$ is not known in general and that makes it hard to avoid some sort of genericity condition. Also some condition on $\lambda$ is necessary. For instance, examples are known (e.g. type $A_{3}$ and $\left.B_{2}\right)$ where Weyl modules $\left(H^{N}\left(w_{0} \cdot \lambda\right)\right)$ have nonsimple $G$-socle. The example in [1] of a decomposable cohomology module could likewise be mentioned. In the first statement of Theorem 2.2 a minimum requirement is of course that $\lambda^{w} \in X(T)_{+}$. If $l(w) \leqslant 1$, then this holds for all $\lambda \in X(T)_{+}$and in this case the statement is in fact true, in general (see [2]).

2.4. Let $\lambda \in X(T)_{+}$and $w \in W$. Then by [12, Satz 3.2(2)] we have

$$
\left[\hat{Z}_{n}(\lambda): \hat{L}_{n}\left(\lambda^{w}\right)\right]=\left[\hat{Z}_{n}(w \cdot \lambda): \hat{L}_{n}(w \cdot \lambda)\right]=1 .
$$

Hence Proposition 2.1 shows that for $\lambda$ generic we have for all $y, w \in W$

$$
\left[H^{l(y)}(y \cdot \lambda): L\left(\lambda^{w}\right)\right]=\left[H^{l(w)}(w \cdot \lambda): L\left(\lambda^{w}\right)\right]=1
$$

(the first equality is a consequence of the fact that $\sum_{i}(-1)^{i} \operatorname{ch} H^{i}(y \cdot \mu)$ is independent of $y \in W$ for all $\mu \in X(T)$ ).

We shall now use this to prove

Proposition. Let $n=1$ and let $\lambda \in X(T)_{+}$be generic. Then for all $y, w \in W$ we have

$$
\operatorname{Hom}_{G}\left(H^{l(y)}(y \cdot \lambda), H^{l(w)}(w \cdot \lambda)\right)=k .
$$

More precisely, the G-homomorphism $T_{w y^{-1}}: H^{l(y)}(y \cdot \lambda) \rightarrow H^{l(w)}(w \cdot \lambda)($ see 4.3 in [4]) is nonzero and up to scalar the only such homomorphism.

Proof. Observe first that if $M$ is a $G$-module with simple socle $L$, then for any $G$-module $N$ we have $\operatorname{dim} \operatorname{Hom}_{G}(N, M) \leqslant[N: L]$. This observation combined with (2) shows that we only have to prove that $T_{w y^{-1}} \neq 0$.

Let $w_{0}=s_{\beta_{1}} \cdots s_{\beta_{N}}$ be a reduced expression for $w_{0}$ such that $s_{\beta_{i}} \cdots s_{\beta_{N}}=w y^{-1}$ for some $i$. Then the homomorphism $T_{w_{0}}: H^{l(y)}(y \cdot \lambda) \rightarrow H^{N-l(y)}\left(w_{0} y \cdot \lambda\right)$ factors through $T_{w y^{-1}}: H^{l(y)}(y \cdot \lambda) \rightarrow H^{l(w)}(w \cdot \lambda)$. As $T_{w_{0}}$ is independent of the reduced expression $[4,4.3]$ we also have the following commutative diagram

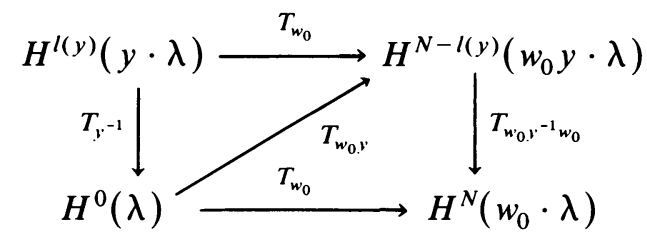


By $[4,4.4]$ we know that $T_{y^{-1}}: H^{l(y)}(y \cdot \lambda) \rightarrow H^{0}(\lambda)$ is nonzero. Hence by Theorem 2.2 we have $\left[\operatorname{Im} T_{y^{-1}}: L\left(\lambda^{w_{0} y}\right)\right] \neq 0$. If $T_{w_{0} y} \neq 0$, then (again by Theorem 2.2) we see that $\left[\operatorname{Im} T_{w_{0} y}: L\left(\lambda^{w_{0} y}\right)\right] \neq 0$. The multiplicity 1 statement in (2) therefore implies that $\left[\operatorname{Im}\left(T_{w_{0} y} \circ T_{y^{-1}}\right): L\left(\lambda^{w_{0} y}\right)\right] \neq 0$. Thus it suffices to prove that $T_{w_{0} y} \neq 0$. The right-hand side of the above diagram shows that it even suffices to verify that $T_{w_{0}}: H^{0}(\lambda) \rightarrow$ $H^{N}\left(w_{0} \cdot \lambda\right)$ is nonzero. But its kernel is $H^{0}(\lambda)^{1}$ so we want to show $H^{0}(\lambda)_{0}=$ $H^{0}(\lambda) / H^{0}(\lambda)^{1} \neq 0$. By [4, Proposition 4.6(i)] we have $H^{0}(\lambda)_{0} \simeq H^{N}\left(w_{0} \cdot \lambda\right)_{m}$, where $m=\sum_{\beta \in R_{+}}\left(1-\nu_{p}\left(\left\langle\beta^{v}, \lambda+\rho\right\rangle\right)\right)$ and Bemerkung 1 in 6.8 of [12] tells us that $H^{N}\left(w_{0} \cdot \lambda\right)_{m} \neq 0$.

REMARK. Only the last line of the proof uses that $n=1$. It seems likely that the proposition holds for all $n$.

2.5. In [3] we gave a formula for the character of the image of the natural map $H^{l\left(s_{\alpha} y\right)}\left(s_{\alpha} y \cdot \lambda\right) \rightarrow H^{l(y)}(y \cdot \lambda)$ for $y \in W, \alpha \in S$ and $\lambda$ "generic" in the lowest $p^{2}$-alcove (we only considered the case where $l\left(s_{\alpha} y\right)>l(y)$ ). We shall now give another proof of this result and thereby characterize the image in a way which allows us in $\$ 4$ to compare it with the image of a homomorphism between cohomology modules of the same degree.

Let $n=1$ and let $\lambda \in X(T)_{+}$be generic. Following Humphreys [10] we define

$$
C_{\alpha}(\lambda)=\sum \chi\left(s_{\alpha} \cdot \lambda+m p \alpha\right) \text { for } \alpha \in R_{+} .
$$

Here the sum runs over all $m$ with $0<m p\left\langle\left\langle\alpha^{v}, \lambda+\rho\right\rangle\right.$ and $\chi(\mu)=$ $\Sigma(-1)^{i} \operatorname{ch} H^{i}(\mu)$ for $\mu \in X(T)$.

If $\alpha \in S$ we let $P_{\alpha}$ denote the minimal parabolic subgroup containing $B$ associated with $\alpha$. We write $H_{\alpha}^{i}(-)=H^{i}\left(P_{\alpha} / B,-\right)$ and if $\mu \in X(T)$ with $\left\langle\alpha^{v}, \mu\right\rangle \geqslant 0$ we set $L_{\alpha}(\mu)$ equal to the irreducible $P_{\alpha}$-module with highest weight $\mu$.

With this notation we have

Proposition. Let $n=1$ and let $\lambda$ be generic. Suppose $w \in W, \alpha \in S$ such that $p$ does not divide $\left\langle\alpha^{v}, w(\lambda+\rho)\right\rangle$. Then the image of the natural homomorphism $H^{l\left(s_{\alpha} w\right)}\left(s_{\alpha} w \cdot \lambda\right) \rightarrow H^{l(w)}(w \cdot \lambda)$ equals $H^{l(w)}\left(L_{\alpha}(w \cdot \lambda)\right)$ if $w^{-1}(\alpha) \in R_{+}$, respectively $H^{l\left(s_{\alpha} w\right)}\left(L_{\alpha}(w \cdot \lambda+a p \alpha)\right)$, where $a=\max \left\{m \mid m p<\left\langle\alpha^{v}, s_{\alpha} w(\lambda+\rho)\right\rangle\right\}$ if $w^{-1}(\alpha) \in R_{-}$. The formal character of the image is $\chi(\lambda)-C_{w^{-1}(\alpha)}(\lambda)$ if $w^{-1}(\alpha) \in R_{+}$, respectively $C_{-w^{-1}(\alpha)}(\lambda)$ if $w^{-1}(\alpha) \in R_{-}$.

Proof. Consider first the case where $w^{-1}(\alpha) \in R_{+}$, i.e. $\left\langle\alpha^{v}, w(\lambda+\rho)\right\rangle=$ $\left\langle w^{-1}(\alpha)^{v}, \lambda+\rho\right\rangle>0$. Then the homomorphism $H^{l\left(s_{\alpha} w\right)}\left(s_{\alpha} w \cdot \lambda\right) \rightarrow H^{l(w)}(w \cdot \lambda)$ is obtained by applying $H^{l(w)}(-)$ to the $P_{\alpha}$-homomorphism $H_{\alpha}^{1}\left(s_{\alpha} w \cdot \lambda\right) \rightarrow H_{\alpha}^{0}(w \cdot \lambda)$. This last homomorphism gives rise to the following two short exact sequences of $P_{\alpha}$-modules

$$
\begin{aligned}
& 0 \rightarrow L_{\alpha}\left(\lambda^{\prime}\right) \rightarrow H_{\alpha}^{1}\left(s_{\alpha} w \cdot \lambda\right) \rightarrow L_{\alpha}(w \cdot \lambda) \rightarrow 0, \\
& 0 \rightarrow L_{\alpha}(w \cdot \lambda) \rightarrow H_{\alpha}^{0}(w \cdot \lambda) \rightarrow L_{\alpha}\left(\lambda^{\prime}\right) \rightarrow 0,
\end{aligned}
$$

where $\lambda^{\prime}=s_{\alpha} w \cdot \lambda+b p \alpha, b=\max \left\{m \mid m p<\left\langle w^{-1}(\alpha)^{v}, \lambda+\rho\right\rangle\right\}$. As $\lambda$ is generic we have by Proposition 2.1

$$
H^{i}\left(H_{\alpha}^{1}\left(s_{\alpha} w \cdot \lambda\right)\right) \simeq H^{i+1}\left(s_{\alpha} w \cdot \lambda\right)=0 \text { for } i \neq l(w)
$$


and

$$
H^{i}\left(H_{\alpha}^{0}(w \cdot \lambda)\right) \simeq H^{i}(w \cdot \lambda)=0 \text { for } i \neq l(w) .
$$

Hence the first sequence in (1) gives

$$
H^{i}\left(L_{\alpha}(w \cdot \lambda)\right) \simeq H^{i+1}\left(L_{\alpha}\left(\lambda^{\prime}\right)\right) \text { for } i \neq l(w), l(w)-1
$$

and we have the exact sequence

$$
\begin{aligned}
0 \rightarrow & H^{l(w)-1}\left(L_{\alpha}(w \cdot \lambda)\right) \rightarrow H^{l(w)}\left(L_{\alpha}\left(\lambda^{\prime}\right)\right) \rightarrow H^{l\left(s_{\alpha} w\right)}\left(s_{\alpha} w \cdot \lambda\right) \\
& \rightarrow H^{l(w)}\left(L_{\alpha}(w \cdot \lambda)\right) \rightarrow H^{l(w)+1}\left(L_{\alpha}\left(\lambda^{\prime}\right)\right) \rightarrow 0 .
\end{aligned}
$$

Similarly, the second sequence in (1) gives

$$
H^{i}\left(L_{\alpha}\left(\lambda^{\prime}\right)\right) \simeq H^{i+1}\left(L_{\alpha}(w \cdot \lambda)\right) \text { for } i \neq l(w), l(w)-1
$$

and the exact sequence

$$
\begin{aligned}
0 \rightarrow & H^{l(w)-1}\left(L_{\alpha}\left(\lambda^{\prime}\right)\right) \rightarrow H^{l(w)}\left(L_{\alpha}(w \cdot \lambda)\right) \rightarrow H^{l(w)}(w \cdot \lambda) \\
& \rightarrow H^{l(w)}\left(L_{\alpha}\left(\lambda^{\prime}\right)\right) \rightarrow H^{l(w)+1}\left(L_{\alpha}(w \cdot \lambda)\right) \rightarrow 0 .
\end{aligned}
$$

Combining (2) and (4) we find

$$
H^{i}\left(L_{\alpha}(w \cdot \lambda)\right) \simeq H^{i+2}\left(L_{\alpha}(w \cdot \lambda)\right) \simeq H^{i+4}\left(L_{\alpha}(w \cdot \lambda)\right) \simeq \cdots \quad \text { for } i>l(w),
$$

respectively

$$
H^{i}\left(L_{\alpha}(w \cdot \lambda)\right) \simeq H^{i-2}\left(L_{\alpha}(w \cdot \lambda)\right) \simeq H^{i-4}\left(L_{\alpha}(w \cdot \lambda)\right) \simeq \cdots \quad \text { for } i<l(w),
$$

i.e. $H^{i}\left(L_{\alpha}(w \cdot \lambda)\right)=0$ for $i \neq l(w)$. Hence also $H^{i}\left(L_{\alpha}\left(\lambda^{\prime}\right)\right)=0$ for $i \neq l(w)$ and (3), respectively (5), reduces to the short exact sequence

$$
0 \rightarrow H^{l(w)}\left(L_{\alpha}\left(\lambda^{\prime}\right)\right) \rightarrow H^{l\left(s_{\alpha} w\right)}\left(s_{\alpha} w \cdot \lambda\right) \rightarrow H^{l(w)}\left(L_{\alpha}(w \cdot \lambda)\right) \rightarrow 0,
$$

respectively

$$
0 \rightarrow H^{l(w)}\left(L_{\alpha}(w \cdot \lambda)\right) \rightarrow H^{l(w)}(w \cdot \lambda) \rightarrow H^{l(w)}\left(L_{\alpha}\left(\lambda^{\prime}\right)\right) \rightarrow 0 .
$$

This shows that the image of $H^{l\left(s_{\alpha} w\right)}\left(s_{\alpha} w \cdot \lambda\right) \rightarrow H^{l(w)}(w \cdot \lambda)$ is indeed equal to $H^{l(w)}\left(L_{\alpha}(w \cdot \lambda)\right)$ and also that

$$
\operatorname{ch} H^{l(w)}\left(L_{\alpha}(w \cdot \lambda)\right)=(-1)^{l(w)} \sum_{i}(-1)^{i} \operatorname{ch} H^{i}\left(L_{\alpha}(w \cdot \lambda)\right)=(-1)^{l(w)} \sum \chi(\nu),
$$

where the last sum runs over the set of weights of $L_{\alpha}(w \cdot \lambda)$. But these weights are $s_{\alpha} w \cdot \lambda+i \alpha$ with $i$ such that the binomial coefficient $\left(\begin{array}{c}\left\langle\alpha^{0}, w \cdot \lambda\right\rangle \\ i\end{array}\right)$ is not divisible by $p$. Since $\chi\left(s_{\alpha} w \cdot \lambda+i \alpha\right)=-\chi(w \cdot \lambda-i \alpha)$ it is easy to see that we have

$$
\begin{aligned}
(-1)^{l(w)} \sum \chi(\nu) & =(-1)^{l(w)} \sum_{m=0}^{b} \chi(w \cdot \lambda-m p \alpha) \\
& =\sum_{m=0}^{b} \chi\left(\lambda-m p w^{-1}(\alpha)\right)=\chi(\lambda)-C_{w^{-1}(\alpha)}(\lambda) .
\end{aligned}
$$

This proves the proposition in the case where $w^{-1}(\alpha) \in R_{+}$. If $w^{-1}(\alpha) \in R_{-}$we use the short exact $P_{\alpha}$-sequences associated to the homomorphism $H_{\alpha}^{0}\left(s_{\alpha} w \cdot \lambda\right) \rightarrow$ $H_{\alpha}^{1}(w \cdot \lambda)$ and proceed exactly as above. Alternatively one may use the fact that the 
sequence

$$
H^{l(w)}(w \cdot \lambda) \rightarrow H^{l\left(s_{\alpha} w\right)}\left(s_{\alpha} w \cdot \lambda\right) \rightarrow H^{l(w)}(w \cdot \lambda)
$$

is exact (see [4, Proposition 4.1]).

2.6. Recall that $\nu \in X(T)$ is called $p$-regular if $p$ does not divide $\left\langle\alpha^{v}, \nu+\rho\right\rangle$ for $\alpha \in R$.

COROllaRy. Let $n=1$ and let $\lambda \in X(T)_{+}$be generic and p-regular. Then for all $\alpha \in R_{+}, w \in W$ we have

$$
\left[C_{\alpha}(\lambda): L\left(\lambda^{w}\right)\right]= \begin{cases}1 & \text { if } w(\alpha) \in R_{-} \\ 0 & \text { otherwise. }\end{cases}
$$

Proof. There exists $\beta \in S, y \in W$ such that $\alpha=y^{-1}(\beta)$. By Proposition 2.4 and Theorem 2.2 the simple module $L\left(\lambda^{w}\right)=\operatorname{Hd}_{G} H^{N-l(w)}\left(w_{0} w \cdot \lambda\right)$ is a composition factor of the image of the natural homomorphism $T_{s_{\beta} y w^{-1} w_{0}}: H^{l\left(w_{0} w\right)}\left(w_{0} w \cdot \lambda\right) \rightarrow$ $H^{l\left(s_{\beta} y\right)}\left(s_{\beta} y \cdot \lambda\right)$. If we compose this homomorphism with $T_{s_{\beta}}: H^{l\left(s_{\beta} y\right)}\left(s_{\beta} y \cdot \lambda\right) \rightarrow$ $H^{l(y)}(y \cdot \lambda)$, then we get the zero map if and only if $l\left(y w^{-1} w_{0}\right)<l\left(s_{\beta} y w^{-1} w_{0}\right)$, i.e. $l\left(s_{\beta} y w^{-1}\right)<l\left(y w^{-1}\right)$ or $w y^{-1}(\beta)=w(\alpha) \in R_{-}$(see [4, 4.1 and 4.3]). Hence $L\left(\lambda^{w}\right)$ is a composition factor of the image of $T_{s_{\beta}}$ if and only if $w(\alpha) \in R_{+}$. Conclusion by Proposition 2.5.

2.7. In [4] we constructed filtrations of certain subquotients of the cohomology modules $H^{*}(\mu), \mu \in X(T)$. For $\lambda$ generic this gives filtrations of all the modules $H^{l(y)}(y \cdot \lambda), y \in W$. As in [4] we denote the filtration levels by subscripts.

Proposition. Let $n=1$ and let $\lambda$ be generic and p-regular. Then for all $y, w \in W$ we have

$$
\left[H^{\prime(y)}(y \cdot \lambda)_{j}: L\left(\lambda^{w}\right)\right]= \begin{cases}1 & \text { if } j=\left|\left\{\alpha \in R_{+} \mid \operatorname{sign} y(\alpha)=\operatorname{sign} w(\alpha)\right\}\right|, \\ 0 & \text { otherwise. }\end{cases}
$$

In particular $\left[H^{N}\left(w_{0} \cdot \lambda\right)_{j}: L\left(\lambda^{w}\right)\right]=\left[H^{0}(\lambda)_{N-j}: L\left(\lambda^{w}\right)\right] \neq 0$ if and only if $j=l(w)$.

Proof. The sum formula [4, Theorem 4.10] takes by Proposition 2.1 the "generic" form

$$
\sum_{j \geqslant 1} \operatorname{ch} H^{l(y)}(y \cdot \lambda)^{j}=\sum_{\alpha \in R_{y}} C_{\alpha}(\lambda)+\sum_{\alpha \in R_{+} \backslash R_{y}}\left(\chi(\lambda)-C_{\alpha}(\lambda)\right),
$$

where we have set $R_{y}=\left\{\alpha \in R_{+} \mid y(\alpha) \in R_{-}\right\}$. Hence by Corollary 2.6 we get

$$
\sum_{j \geqslant 1}\left[H^{l(y)}(y \cdot \lambda)^{j}: L\left(\lambda^{w}\right)\right]=\left|R_{y} \cap R_{w}\right|+\left|R_{+} \backslash\left(R_{y} \cup R_{w}\right)\right| .
$$

As the multiplicity of $L\left(\lambda^{w}\right)$ in $H^{l(y)}(y \cdot \lambda)$ is 1 this proves the proposition.

2.8. Let $y, w \in W$ and $\beta \in S$. Then

$$
\begin{aligned}
\mid\{\alpha & \left.\in R_{+} \mid \operatorname{sign}\left(s_{\beta} y(\alpha)\right)=\operatorname{sign} w(\alpha)\right\} \mid \\
& =\left|\left\{\alpha \in R_{+} \mid \operatorname{sign} y(\alpha)=\operatorname{sign} w(\alpha)\right\}\right|-\operatorname{sign} w y^{-1}(\beta) .
\end{aligned}
$$


Indeed, using the notation $R_{y}$ from the proof of Proposition 2.7 we have that if $y^{-1}(\beta) \in R_{+}$, then $R_{s_{\beta} y}=R_{y} \cup\left\{y^{-1}(\beta)\right\}$ and hence

$$
\begin{aligned}
&\left(R_{s_{\beta} y} \cap R_{w}\right) \cup\left(R_{+} \backslash\right.\left.\left(R_{s_{\beta} y} \cup R_{w}\right)\right) \\
&=\left\{\begin{array}{r}
\left(R_{y} \cap R_{w}\right) \cup\left(R_{+} \backslash\left(R_{y} \cup R_{w}\right)\right) \cup\left\{y^{-1}(\beta)\right\} \\
\left(R_{y} \cap R_{w}\right) \cup\left(R_{+} \backslash\left(R_{y} \cup R_{w} \cup\left\{y^{-1}(\beta)\right\}\right)\right) \\
\text { if } w y^{-1}(\beta) \in R_{-},
\end{array}\right. \\
& \text {if } w y^{-1}(\beta) \in R_{+} .
\end{aligned}
$$

The case $y^{-1}(\beta) \in R_{-}$follows by symmetry.

By Proposition 2.5 and Corollary 2.6 we see that $L\left(\lambda^{w}\right)$ is a composition factor of the image of $T_{s_{\beta}}: H^{l\left(s_{\beta} y\right)}\left(s_{\beta} y \cdot \lambda\right) \rightarrow H^{l(y)}(y \cdot \lambda)$ if and only if $w y^{-1}(\beta) \in R_{+}$. Hence combining (1) with Proposition 2.7 we obtain

COROLlaRY. Let $n=1$ and let $\lambda$ be generic and p-regular. Then for all $y, w \in W$ and $\beta \in S$ we have

$L\left(\lambda^{w}\right)$ is a composition factor of the image of the natural map $H^{l\left(s_{\beta} y\right)}\left(s_{\beta} y \cdot \lambda\right) \rightarrow H^{l(y)}(y \cdot \lambda)$ if and only if the filtration level of $L\left(\lambda^{w}\right)$ in $H^{l(y)}(y \cdot \lambda)$ is 1 higher than in $H^{l\left(s_{\beta} y\right)}\left(s_{\beta} y \cdot \lambda\right)$.

REMARK. (a) This corollary shows that Humphreys' conjecture [10, Conjecture $2.4(2)]$ holds for the "extremal" composition factors $L\left(\lambda^{w}\right)$ of $H^{l(y)}(y \cdot \lambda), w$, $y \in W$.

(b) In the multiplicity free case, Proposition 2.5 allows us to determine the composites of the homomorphisms between the cohomology modules $H^{l(w)}(w \cdot \lambda)$, $w \in W$. This was illustrated for type $A_{2}$ and $B_{2}$ at the end of [3]. As was pointed out to me by J. E. Humphreys some of the numbers in Figures 2(a)-(d) are, however, not correctly placed (some of the 2's and 3's have been interchanged and in Figure 2(c) there is a missing alcove number).

3. Extensions. We shall now explore the relation between $G_{n} B$-extensions of simple modules and the corresponding extensions between simple $G$-modules. The idea is to use the result of Theorem 2.2 to see that certain $G_{n} B$-extensions give rise to nonsplit $G$-extensions via the functors $H^{i}\left(G / G_{n} B,-\right), i \geqslant 0$.

3.1. Recall that if $H^{\prime}$ is a closed subgroup scheme of a group scheme $H$ and $E$ (resp. $\left.E^{\prime}\right)$ is a representation of $H\left(\right.$ resp. $H^{\prime}$ ), then we have a spectral sequence

$$
\operatorname{Ext}_{H}^{i}\left(E, H^{j}\left(H / H^{\prime}, E^{\prime}\right)\right) \Rightarrow \operatorname{Ext}_{H^{\prime}}^{i+j}\left(E, E^{\prime}\right) .
$$

Also if $H^{\prime}$ is normal in $H$, then we have the Lyndon-Hochschild-Serre spectral sequence

$$
\operatorname{Ext}_{H / H^{\prime}}^{i}\left(A, \operatorname{Ext}_{H^{\prime}}^{j}(B, C)\right) \Rightarrow \operatorname{Ext}_{H}^{i+j}(A \otimes B, C)
$$


for any $H$-modules $B, C$ with $B$ finite dimensional and any $H / H^{\prime}$-module $A$. In particular, in this case we have the 5-term exact sequence

(3)

$$
\begin{aligned}
0 \rightarrow & \operatorname{Ext}_{H / H^{\prime}}^{1}\left(A, \operatorname{Hom}_{H^{\prime}}(B, C)\right) \rightarrow \operatorname{Ext}_{H}^{1}(A \otimes B, C) \rightarrow \operatorname{Hom}_{H / H^{\prime}}\left(A, \operatorname{Ext}_{H^{\prime}}^{1}(B, C)\right) \\
& \rightarrow \operatorname{Ext}_{H / H^{\prime}}^{2}\left(A, \operatorname{Hom}_{H^{\prime}}(B, C)\right) \rightarrow \operatorname{Ext}_{H}^{2}(A \otimes B, C) .
\end{aligned}
$$

3.2. Lemma. Let $\lambda, \mu \in X(T)$. Then

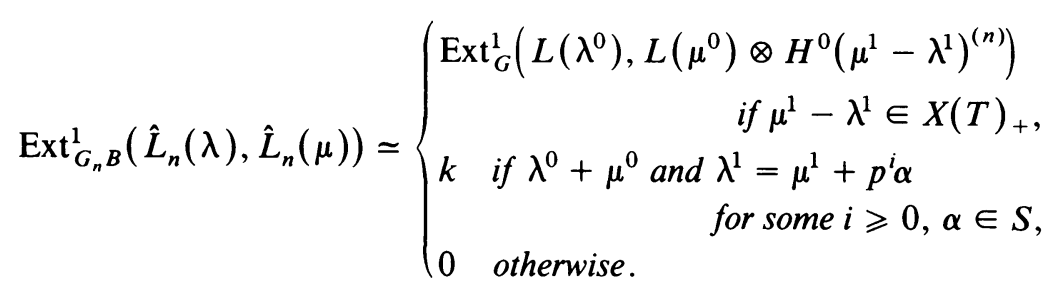

Proof. If $\mu^{1}-\lambda^{1} \in X(T)_{+}$, then $H^{i}\left(\mu^{1}-\lambda^{1}\right)=0$ for $i>0$ by Kempf's vanishing theorem. Hence by $3.1(1)$ taken relative to $G_{n} B \leqslant G$ and using 1.6(2) we have for all $i$

$$
\begin{aligned}
\operatorname{Ext}_{G_{n} B}^{i}\left(\hat{L}_{n}(\lambda), \hat{L}_{n}(\mu)\right) & \simeq \operatorname{Ext}_{G_{n} B}^{i}\left(L\left(\lambda^{0}\right), L\left(\mu^{0}\right) \otimes p^{n}\left(\mu^{1}-\lambda^{1}\right)\right) \\
& \simeq \operatorname{Ext}_{G}^{i}\left(L\left(\lambda^{0}\right), L\left(\mu^{0}\right) \otimes H^{0}\left(\mu^{1}-\lambda^{1}\right)^{(n)}\right) .
\end{aligned}
$$

On the other hand if $\mu^{1}-\lambda^{1} \notin X(T)_{+}$, then $H^{0}\left(\mu^{1}-\lambda^{1}\right)=0$; the same reasoning gives

$$
\begin{aligned}
\operatorname{Ext}_{G_{n} B}^{1}\left(\hat{L}_{n}(\lambda), \hat{L}_{n}(\mu)\right) & \simeq \operatorname{Hom}_{G}\left(L\left(\lambda^{0}\right), L\left(\mu^{0}\right) \otimes H^{1}\left(\mu^{1}-\lambda^{1}\right)^{(n)}\right) \\
& \simeq\left(\operatorname{Hom}_{G_{n}}\left(L\left(\lambda^{0}\right), L\left(\mu^{0}\right)\right) \otimes H^{1}\left(\mu^{1}-\lambda^{1}\right)^{(n)}\right)^{G / G_{n}} .
\end{aligned}
$$

This is clearly zero unless $\lambda^{0}=\mu^{0}$. Also by [2, Theorem 3.5] $H^{1}\left(\mu^{1}-\lambda^{1}\right)^{G}=0$ for $\mu^{1}-\lambda^{1} \notin-p^{i} S$ and $H^{1}\left(-p^{i} \alpha\right)^{G}=k$ for all $i \geqslant 0, \alpha \in S$. This proves the lemma.

3.3. TheOREM. Let $\lambda, \mu \in X(T)_{+}$with $\mu$ generic. Assume that $\lambda^{0} \neq \mu^{0}$. Then for all $w \in W$ we have

if $E$ is a nontrivial $G_{n} B$-extension of $\hat{L}_{n}(w \cdot \lambda)$ by $\hat{L}_{n}(w \cdot \mu)$, then $H^{l(w)}\left(G / G_{n} B, E\right)$ is a nontrivial G-extension of $L\left(\lambda^{w}\right)$ by $L\left(\mu^{w}\right)$.

Proof. Let $w \in W$. Note first that $(w \cdot \lambda)^{0} \neq(w \cdot \mu)^{0}$. In fact, $(w \cdot \lambda)^{0}=(w \cdot \mu)^{0}$ if and only if $p^{n}\left(\lambda_{w}^{0}-\mu_{w}^{0}\right)=w \cdot \mu^{0}-w \cdot \lambda^{0}=w\left(\mu^{0}-\lambda^{0}\right)$. As $\mu^{0}, \lambda^{0} \in X_{n}(T)$ we cannot have $\mu^{0}-\lambda^{0} \in p^{n} X(T)$.

We claim that $\operatorname{Ext}_{G_{n} B}^{1}\left(\hat{L}_{n}(w \cdot \lambda), \hat{Q}_{n}(w \cdot \mu)\right)=0$. As $\hat{Q}_{n}(w \cdot \mu)$ is injective for $G_{n}$ we certainly have $\operatorname{Ext}_{G_{n}}^{1}\left(\hat{L}_{n}(w \cdot \lambda), \hat{Q}_{n}(w \cdot \mu)\right)=0$ and since $(w \cdot \lambda)^{0} \neq(w \cdot \mu)^{0}$ we also have $\operatorname{Hom}_{G_{n}}\left(\hat{L}_{n}(w \cdot \lambda), \hat{Q}_{n}(w \cdot \mu)\right)=0$. The claim therefore follows by 3.1(3) (relative to $G_{n} \triangleleft G_{n} B$ ). 
The vanishing of this Ext-group implies that $E \subset \hat{Q}_{n}(w \cdot \mu)$. As in the proof of Theorem 2.2 we have $H^{i}\left(G / G_{n} B, \hat{L}_{n}(\nu)\right)=0$ for $i \neq l(w)$ and $\hat{L}_{n}(\nu)$ a composition factor of $\hat{Q}_{n}(w \cdot \mu)$. Hence the inclusion $E \subset \hat{Q}_{n}(w \cdot \mu)$ gives rise to an inclusion

$$
\begin{aligned}
H^{l(w)}\left(G / G_{n} B, E\right) & \subset H^{l(w)}\left(G / G_{n} B, \hat{Q}_{n}(w \cdot \mu)\right) \\
& \simeq Q_{n}\left((w \cdot \mu)^{0}\right) \otimes H^{l(w)}\left((w \cdot \mu)^{1}\right)^{(n)} .
\end{aligned}
$$

The latter has simple $G$-socle (namely $L\left(\mu^{w}\right)$ ) and hence so has $H^{l(w)}\left(G / G_{n} B, E\right)$. Note finally that

$$
H^{l(w)}\left(G / G_{n} B, \hat{L}_{n}(w \cdot \lambda)\right) \simeq L\left(\lambda^{w}\right)
$$

so that $H^{(w)}\left(G / G_{n} B, E\right) / L\left(\mu^{w}\right) \simeq L\left(\lambda^{w}\right)$.

REMARK. By Lemma 3.2 the only $G_{n} B$-extensions of $\hat{L}_{n}(\lambda)$ by $\hat{L}_{n}(\mu)$ with $\lambda^{0}=\mu^{0}$ are those where $\lambda^{1}=\mu^{1}+p^{i} \alpha, i \geqslant 0, \alpha \in S$. These do not always give rise to $G$-extensions. For if $\mu \in X(T)_{+}$is generic and $E$ is a $G_{n} B$-extension of the type mentioned, then applying $H^{0}\left(G / G_{n} B,-\right)$ we obtain the short exact sequence

$$
0 \rightarrow L\left(\mu^{0}\right) \otimes H^{0}\left(\mu^{1}\right)^{(n)} \rightarrow H^{0}\left(G / G_{n} B, E\right) \rightarrow L\left(\mu^{0}\right) \otimes H^{0}\left(\lambda^{1}\right) \rightarrow 0
$$

and by 3.1(3) relative to $G_{n} \triangleleft G$ and [5, Theorem 4.5] we have

$$
\operatorname{Ext}_{G}^{1}\left(L\left(\mu^{0}\right) \otimes H^{0}\left(\mu^{1}\right)^{(n)}, L\left(\mu^{0}\right) \otimes H^{0}\left(\lambda^{1}\right)^{(n)}\right) \simeq \operatorname{Ext}_{G}^{1}\left(H^{0}\left(\mu^{1}\right), H^{0}\left(\lambda^{1}\right)\right) .
$$

If both $\lambda$ and $\mu$ are generic (by the above this means $\lambda^{1}=\mu^{1}+\alpha$ for some $\alpha \in S$ ), then $\mu^{1}, \lambda^{1} \in C_{1}$ and hence both $H^{0}\left(\lambda^{1}\right)$ and $H^{0}\left(\mu^{1}\right)$ are irreducible. Therefore $\operatorname{Ext}_{G}^{1}\left(H^{0}\left(\mu^{1}\right), H^{0}\left(\lambda^{1}\right)\right)=0$ and the sequence above splits.

3.4. Corollary. Let $\lambda, \mu \in X(T)_{+}$with $\lambda^{0} \neq \mu^{0}$. Then for all $w \in W$ for which $\lambda^{1}+w\left(\mu^{1}\right)$ lies in $C_{1}$ far from the walls we have

$$
\operatorname{Ext}_{G}^{1}\left(L\left(\lambda^{0}\right), L\left(\mu^{0}\right) \otimes H^{0}\left(\mu^{1}\right)^{(n)}\right) \subset \operatorname{Ext}_{G}^{1}\left(L(\lambda), L\left(\mu^{0}+p^{n}\left(\lambda^{1}+w\left(\mu^{1}\right)\right)\right)\right) .
$$

Proof. From Lemma 3.2 we know that

$$
\operatorname{Ext}_{G}^{1}\left(L\left(\lambda^{0}\right), L\left(\mu^{0}\right) \otimes H\left(\mu^{1}\right)^{(n)}\right) \simeq \operatorname{Ext}_{G_{n} B}^{1}\left(L\left(\lambda^{0}\right), \hat{L}_{n}(\mu)\right) .
$$

Of course this is also isomorphic to $\operatorname{Ext}_{G_{n} B}^{1}\left(\hat{L}_{n}\left(\lambda^{0}+p^{n} w^{-1} \cdot \lambda^{1}\right), \hat{L}_{n}\left(\mu+p^{n} w^{-1} \cdot \lambda^{1}\right)\right)$ and by Theorem $3.3 H^{\prime(w)}\left(G / G_{n} B,-\right)$ embeds this Ext-group into

$$
\operatorname{Ext}_{G}^{1}\left(L(\lambda), L\left(\mu^{0}+p^{n}\left(\lambda^{1}+w\left(\mu^{1}\right)\right)\right)\right) .
$$

REMARK. In 3.6 we shall prove a result which gives an explanation for and a more precise statement about the relation between the Ext-groups occurring in this corollary. We leave to the reader the task of checking exactly what the condition $\lambda^{1}+w\left(\mu^{1}\right)$ being far from the walls of $C_{1}$ shall mean. The corollary was the first evidence we had for the result in Theorem 3.6 below.

3.5. Let $\lambda, \mu \in X_{n}(T)$ and let $E$ be the $G$-module determined by $E^{(n)}=$ $\operatorname{Ext}_{G_{n}}^{1}(L(\lambda), L(\mu))$. Then

LEMMA. If $\nu$ is a weight of $E$, then $\left|\left\langle\alpha^{v}, \nu\right\rangle\right|<2(h-1)$ for all $\alpha \in R$.

Proof. To see this let $R$ be indecomposable. Recall that if $\nu$ is a weight of $E$, then $\lambda+p^{n} \nu \leqslant 2\left(p^{n}-1\right) \rho+w_{0}(\mu)$. Hence if $w \in W$ such that $w(\nu) \in X(T)_{+}$, then

$$
\max _{\alpha \in R}\left|\left\langle\alpha^{v}, \nu\right\rangle\right|=\max _{\alpha \in R}\left|\left\langle\alpha^{v}, w(\nu)\right\rangle\right|=\left\langle\alpha_{0}^{v}, w(\nu)\right\rangle
$$


and by the above inequality we get $\left\langle\alpha_{0}^{v}, \lambda\right\rangle+p^{n}\left\langle\alpha_{0}^{v}, w(\nu)\right\rangle \leqslant 2\left(p^{n}-1\right)(h-1)+$ $\left\langle\alpha_{0}^{v}, w_{0}(\mu)\right\rangle \leqslant 2\left(p^{n}-1\right)(h-1)$ and hence $\left\langle\alpha_{0}^{v}, w(\nu)\right\rangle<2(h-1)$.

3.6. THEOREM. Let $\lambda \in X(T)_{+}$and suppose $2(h-1) \leqslant\left\langle\alpha^{v}, \lambda^{1}\right\rangle \leqslant p-2(h-1)$ for all $\alpha \in R_{+}$. Then for all $\mu \in X(T)_{+}$we have

(i) $\operatorname{dim} \operatorname{Ext}_{G}^{1}\left(L\left(\lambda^{0}\right), L\left(\mu^{0}\right) \otimes H^{0}\left(\mu^{1}\right)^{(n)}\right)=\left[\operatorname{Ext}_{G_{n}}^{1}\left(L\left(\lambda^{0}\right), L\left(\mu^{0}\right)\right)^{*}: L\left(\mu^{1}\right)^{(n)}\right]$,

(ii) $\operatorname{dim} \operatorname{Ext}_{G}^{1}\left(L(\lambda), L\left(\mu^{0}+p^{n}\left(\lambda^{1}+w\left(\mu^{1}\right)\right)\right)\right)=\operatorname{dim}_{\operatorname{Ext}_{G_{n}}^{1}}\left(L\left(\lambda^{0}\right), L\left(\mu^{0}\right)\right)_{p^{n} \mu^{1}}^{*}$ for all $w$ when $\lambda^{0} \neq \mu^{0}$. In particular the left-hand side is independent of $w$ and of $\lambda^{1}$ (in the prescribed region).

Proof. (i) If $\lambda^{0}=\mu^{0}$, then both sides are 0 by [5, Theorem 4.5]. So suppose $\lambda^{0} \neq \mu^{0}$. Then by $3.1(3)$ (relative to $G_{n} \triangleleft G$ ) we have

$$
\operatorname{Ext}_{G}^{1}\left(L\left(\lambda^{0}\right), L\left(\mu^{0}\right) \otimes H^{0}\left(\mu^{1}\right)^{(n)}\right) \simeq \operatorname{Hom}_{G / G_{n}}\left(\operatorname{Ext}_{G_{n}}^{1}\left(L\left(\lambda^{0}\right), L\left(\mu^{0}\right)\right)^{*}, H^{0}\left(\mu^{1}\right)^{(n)}\right) .
$$

Our assumption implies that $p>4(h-1)$ so by [5, Proposition 5.5] we know that $\operatorname{Ext}_{G_{n}}^{1}\left(L\left(\lambda^{0}\right), L\left(\mu^{0}\right)\right)$ is completely reducible and (i) follows.

To prove (ii) we first notice that

$$
\begin{aligned}
\operatorname{Ext}_{G}^{1}\left(L(\lambda), L\left(\mu^{0}+p^{n}\left(\lambda^{1}+w\left(\mu^{1}\right)\right)\right)\right) \\
\simeq \operatorname{Hom}_{G / G_{n}}\left(L\left(\lambda^{1}\right)^{(n)} \otimes \operatorname{Ext}_{G_{n}}^{1}\left(L\left(\lambda^{0}\right), L\left(\mu^{0}\right)\right)^{*}, L\left(\lambda^{1}+w\left(\mu^{1}\right)\right)^{(n)}\right) .
\end{aligned}
$$

Using Lemma 3.5 we see that $L\left(\lambda^{1}\right)^{(n)} \otimes \operatorname{Ext}_{G_{n}}^{1}\left(L\left(\lambda^{0}\right), L\left(\mu^{0}\right)\right)^{*} \simeq \oplus L\left(\lambda^{1}+\nu\right)^{(n)}$, where the sum runs over all $\nu$ for which $p^{n} \nu$ is a weight of $\operatorname{Ext}_{G_{n}}{ }_{n}\left(L\left(\lambda^{0}\right), L\left(\mu^{0}\right)\right)^{*}$ and with $L\left(\lambda^{1}+\nu\right)^{(n)}$ occurring with multiplicity equal to the multiplicity of $p^{n} \nu$ as a weight of $\operatorname{Ext}_{G_{n}}^{1}\left(L\left(\lambda^{0}\right), L\left(\mu^{0}\right)\right)^{*}$. Putting this together and using that the set of weights of $\operatorname{Ext}_{G_{n}}^{1}\left(L\left(\lambda^{0}\right), L\left(\mu^{0}\right)\right)$ is $W$-invariant we obtain (ii).

REMARKs. (a) The proof shows that (i) holds for all $\lambda$ as long as $p>3(h-1)$. Note that (i) is a reformulation of $[5,5.5]$.

(b) In (ii) we have

$$
\begin{aligned}
\operatorname{Ext}_{G_{n}}^{1}\left(L\left(\lambda^{0}\right), L\left(\mu^{0}\right)\right)_{p^{n} \mu^{1}}^{*} & \simeq \operatorname{Ext}_{G_{n} T}^{1}\left(L\left(\lambda^{0}\right), \hat{L}_{n}(\mu)\right) \\
& \simeq \operatorname{Ext}_{G_{n} T}^{1}\left(\hat{L}(\lambda), \hat{L}_{n}\left(\mu+p^{n} \lambda^{1}\right)\right)
\end{aligned}
$$

(c) If we combine (i) and (ii) then we obtain another proof of Corollary 3.4. Note also that in this way we see that equality holds in Corollary 3.4 if and only if $p^{n} \mu^{1}$ is a maximal weight in $\operatorname{Ext}_{G_{n}}{ }_{n}\left(L\left(\lambda^{0}\right), L\left(\mu^{0}\right)\right)^{*}$. For type $A_{2}$ all dominant weights of $\operatorname{Ext}_{G_{n}}^{1}\left(L\left(\lambda^{0}\right), L\left(\mu^{0}\right)\right)^{*}$ are maximal, while the same is true for type $B_{2}$ except in some cases for the zero weight.

4. Homomorphisms. In this section we study homomorphisms between cohomology modules of the same degree corresponding to weights in adjacent alcoves. We relate these maps to the natural homomorphisms considered in $\S 2$.

Throughout this section $n=1$.

4.1. Let $w \in W, \beta \in S$ such that $\alpha=w^{-1}(\beta) \in R_{+}$. Let $\lambda \in X(T)$ and assume $a p<\left\langle\alpha^{v}, \lambda+\rho\right\rangle<(a+1) p$. Set $\mu=s_{\alpha} \cdot \lambda+a p \alpha$. 
Lemma. Let $\lambda, w, \alpha$ and $\mu$ be as above. Then $\mu^{w}=\lambda^{w s_{\alpha}}$.

Proof. By definition, $\mu^{0}=p \lambda_{s}^{0}+s_{\alpha} \cdot \lambda^{0}$ and $\mu^{1}=s_{\alpha}\left(\lambda^{1}\right)+a \alpha-\lambda_{s_{\alpha}}^{0}$. Hence $\mu_{w}^{0}$ $=\lambda_{w s_{\alpha}}^{0}-w\left(\lambda_{s_{\alpha}}^{0}\right)$ and

$$
\begin{aligned}
\mu^{w}= & p\left(\lambda_{w s_{\alpha}}^{0}-w_{\odot}\left(\lambda_{s_{\alpha}}^{0}\right)\right)+p w\left(\lambda_{s_{\alpha}}^{0}\right)+w s_{\alpha} \cdot \lambda^{0} \\
& +p\left(s_{\alpha}\left(\lambda^{1}\right)+a \alpha-\lambda_{s_{\alpha}}^{0}+w^{-1} \cdot\left(-\lambda_{w s_{\alpha}}^{0}\right)+\lambda_{s_{\alpha}}^{0}\right) \\
= & p \lambda_{w s_{\alpha}}^{0}+w s_{\alpha} \cdot \lambda^{0}+p\left(\lambda^{1}+\left(a-\left\langle\alpha^{v}, \lambda^{1}\right\rangle\right) \alpha+w^{-1} \cdot\left(-\lambda_{w s_{\alpha}}^{0}\right)\right) .
\end{aligned}
$$

So we are done if we show that $\left(a-\left\langle\alpha^{v}, \lambda^{1}\right\rangle\right) \alpha+w^{-1} \cdot\left(-\lambda_{w s_{\alpha}}^{0}\right)=s_{\alpha^{\prime}} w^{-1} \cdot\left(-\lambda_{w s_{\alpha}}^{0}\right)$, i.e. that $\left\langle\alpha^{v}, w^{-1}\left(\rho-\lambda_{w s_{\alpha}}^{0}\right)\right\rangle=\left\langle\alpha^{v}, \lambda^{1}\right\rangle-a$. However, with the notation from 1.8 we have $a=\left\langle\alpha^{v}, \lambda^{1}\right\rangle+n_{\alpha}^{\lambda^{0}}$ and $\left\langle\alpha^{v}, w^{-1}\left(\rho-\lambda_{w s_{\alpha}}\right)\right\rangle=\left\langle\beta^{v}, \rho-\lambda_{w s_{\alpha}}^{0}\right\rangle=1+n_{-\alpha}^{\lambda^{0}}$. As $n_{-\alpha}^{\lambda^{0}}=-n_{\alpha}^{\lambda^{0}}-1$ we are done.

4.2. Let $\lambda, w, \alpha$ and $\mu$ be as in $\$ 4.1$.

Proposition. Suppose $\lambda$ and $\mu$ are both generic. Then there exists a unique (up to scalar ) homomorphism

$$
\phi: H^{l(w)}(w \cdot \lambda) \rightarrow H^{l(w)}(w \cdot \mu) .
$$

Moreover, $\operatorname{Im} \phi=H^{l(w)}\left(L_{\beta}(w \cdot \mu)\right)$ and $\operatorname{ch} \operatorname{Im} \phi=C_{\alpha}(\lambda)$. The composite

$$
H^{l(y)}(y \cdot \lambda) \stackrel{T_{w y}-1}{\rightarrow} H^{l(w)}(w \cdot \lambda) \stackrel{\phi}{\rightarrow} H^{l(w)}(w \cdot \mu)
$$

is nonzero if and only if $y(\alpha) \in R_{+}$.

Proof. The uniqueness of $\phi$ follows immediately because by Lemma 4.1 and 2.4(2) we have

$$
\left[H^{l(w)}(w \cdot \lambda): \operatorname{Soc} H^{l(w)}(w \cdot \mu)\right]=\left[H^{l(w)}(w \cdot \lambda): L\left(\lambda^{w s_{\alpha}}\right)\right]=1 .
$$

To see that $\phi$ exists note first that we have a homomorphism of $P_{\beta}$-modules $H_{\beta}^{0}(w \cdot \lambda) \rightarrow H_{\beta}^{0}(w \cdot \mu)$ which gives rise to the two short exact sequences

$$
\begin{aligned}
& 0 \rightarrow L_{\beta}(w \cdot \lambda) \rightarrow H_{\beta}^{0}(w \cdot \lambda) \rightarrow L_{\beta}(w \cdot \mu) \rightarrow 0, \\
& 0 \rightarrow L_{\beta}(w \cdot \mu) \rightarrow H_{\beta}^{0}(w \cdot \mu) \rightarrow L_{\beta}(w \cdot(\lambda-p \alpha)) \rightarrow 0 .
\end{aligned}
$$

From the proof of Proposition 2.5 we have that $H^{i}\left(L_{\beta}(w \cdot \lambda)\right)=H^{i}\left(L_{\beta}(w \cdot \mu)\right)=$ $H^{i}\left(L_{\beta}(w \cdot(\lambda-p \alpha))\right)=0$ for $i \neq l(w)$ and hence by applying $H^{l(w)}(-)$ to (1) we obtain a homomorphism

$$
H^{l(w)}(w \cdot \lambda) \rightarrow H^{l(w)}(w \cdot \mu)
$$

whose image is $H^{l(w)}\left(L_{\beta}(w \cdot \mu)\right)$. This establishes the existence of $\phi$ and the statement about its image (compare with the proof of Proposition 2.5 for the computation of the character).

From Corollary 2.6 it follows that $\left[C_{\alpha}(\lambda): L\left(\lambda^{w_{0} y}\right)\right] \neq 0$ if and only if $y(\alpha) \in R_{+}$. Since $\operatorname{Hd} H^{l(y)}(y \cdot \lambda)=L\left(\lambda^{w_{0} y}\right)$ this gives the last statement.

4.3. In the setup of $\$ 4.2$ we also have a homomorphism $H_{\beta}^{1}\left(s_{\beta} w \cdot \mu\right) \rightarrow H_{\beta}^{1}\left(s_{\beta} w \cdot \lambda\right)$ and by similar reasoning this gives rise to a homomorphism (unique up to scalar)

$$
\phi^{\prime}: H^{l(w)+1}\left(s_{\beta} w \cdot \mu\right) \rightarrow H^{l(w)+1}\left(s_{\beta} w \cdot \lambda\right)
$$

with $\operatorname{Im} \phi=H^{l(w)}\left(L_{\beta}(w \cdot \mu)\right)$ and $\operatorname{ch} \operatorname{Im} \phi^{\prime}=C_{\alpha}(\lambda)$. 
Combining this with Propositions 2.5 and 4.2 we obtain the "exact square":

$$
\begin{array}{ccc}
H^{l\left(s_{\beta} w\right)}\left(s_{\beta} w \cdot \lambda\right) & \stackrel{T_{s_{\beta}}}{\rightarrow} & H^{l(w)}(w \cdot \lambda) \\
\phi^{\prime} \uparrow & & \downarrow \phi \\
H^{l\left(s_{\beta} w\right)}\left(s_{\beta} w \cdot \mu\right) & \stackrel{T_{s_{\beta}}}{\leftarrow} & H^{l(w)}(w \cdot \mu)
\end{array}
$$

More generally if we set $\lambda_{i}=\lambda-i p \alpha, \mu_{i}=\mu-i p \alpha$ for $i=1,2, \ldots, r$ and assume that these are all generic, then we can put together the resulting "exact squares" into an "exact rectangle":

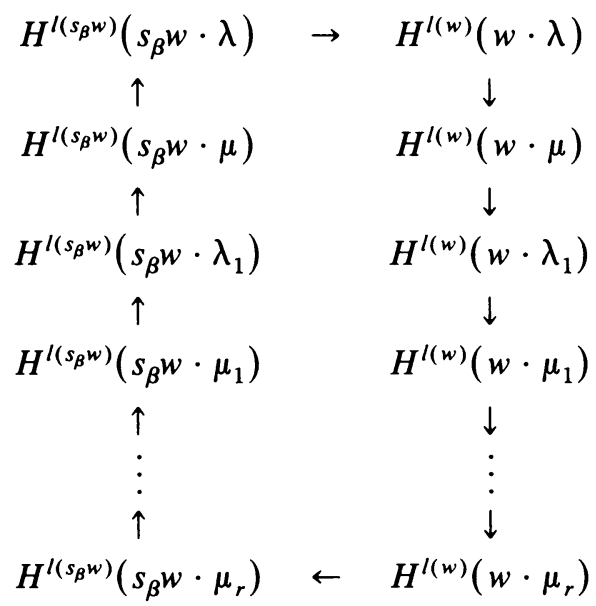

4.4. In the case where $w=1$ the existence and uniqueness of a homomorphism $\phi$ : $H^{0}(\lambda) \rightarrow H^{0}\left(s_{\beta} \cdot \lambda+a p \beta\right)$ is clear for all $\lambda \in X(T)_{+}$and $\beta \in S$ with $0<a p \leqslant$ $\left\langle\beta^{v}, \lambda\right\rangle<(a+1) p-1$. In this case it is shown (by different methods and under somewhat different conditions on $\lambda$ ) in [3, Proposition 4.3] and [12, Satz 3.12] that $\operatorname{ch} \operatorname{Im} \phi=C_{\beta}(\lambda)$. If we examine the proof of Proposition 4.2 we see that the condition we need on $\lambda$ when $w=1$ is only that $H^{i}\left(s_{\beta} \cdot \lambda\right)=0$ for $i>1$. Unfortunately, no general criterion which ensures this is available.

If we consider the case $a=1$ where we have $L_{\beta}\left(s_{\beta} \cdot \lambda+a p \beta\right)=H_{\beta}^{0}\left(s_{\beta} \cdot \lambda+a p \beta\right)$ our method gives the exact sequence

$$
\begin{aligned}
0 & \rightarrow H^{0}\left(s_{\beta} \cdot \lambda+p \beta\right) \rightarrow H^{1}\left(s_{\beta} \cdot \lambda\right) \rightarrow H^{0}(\lambda) \\
& \rightarrow H^{0}\left(s_{\beta} \cdot \lambda+p \beta\right) \rightarrow H^{2}\left(s_{\beta} \cdot \lambda\right) \rightarrow 0 .
\end{aligned}
$$

The rank 2 cases give examples where $H^{2}\left(s_{\beta} \cdot \lambda\right) \neq 0$ and in these cases the equality $\operatorname{ch} \operatorname{Im} \phi=C_{\beta}(\lambda)$ is therefore not valid.

Let us mention that the sequence (1) can be used in combination with Jantzen's work in $[12,6.3]$ to analyse the head of $H^{0}(\lambda)$. One immediate consequence of (1) is

If in the above situation $H^{2}\left(s_{\beta} \cdot \lambda\right)=0$,

$$
\text { then } \operatorname{Hd}\left(H^{0}\left(s_{\beta} \cdot \lambda+p \beta\right)\right) \subset \operatorname{Hd} H^{0}(\lambda) .
$$

Of course it also follows from (1) that $\operatorname{Hd}\left(H^{2}\left(s_{\beta} \cdot \lambda\right)\right) \subset \operatorname{Hd} H^{0}\left(s_{\beta} \cdot \lambda+p \beta\right)$. 
4.5. Let $\lambda, w, \alpha$ and $\mu$ be as in 4.1. Writing $\hat{Z}_{\beta}=\operatorname{Ind}_{B}^{P_{\beta 1} B}$ we have a (unique up to scalar) $P_{\beta 1} B$-homomorphism

$$
\hat{\phi}_{\beta}: \hat{Z}_{\beta}(w \cdot \lambda) \rightarrow \hat{Z}_{\beta}(w \cdot \mu) .
$$

It is easy to see that

$$
\operatorname{ch}\left(\operatorname{Im} \hat{\phi}_{\beta}\right)=\sum_{m \geqslant 0}\left(\operatorname{ch} \hat{Z}_{\beta}(w \cdot \mu-m p \beta)-\operatorname{ch} \hat{Z}_{\beta}(w \cdot \lambda-(m+1) p \beta)\right) .
$$

Inducing further from $P_{\beta 1} B$ to $G_{1} B$ we obtain a $G_{1} B$-homomorphism

$$
\hat{\phi}: \hat{Z}_{1}(w \cdot \lambda) \rightarrow \hat{Z}_{1}(w \cdot \mu)
$$

and since this induction is exact we get from (1)

$$
\operatorname{ch} \operatorname{Im} \hat{\phi}=\sum_{m \geqslant 0}\left(\operatorname{ch} \hat{Z}_{1}(w \cdot \mu-m p \beta)-\operatorname{ch} \hat{Z}_{1}(w \cdot \lambda-(m+1) p \beta)\right) .
$$

For each composition factor $\hat{L}_{1}(\nu)$ of $\operatorname{Im} \hat{\phi}$ it is clear that

$$
\left[\hat{Z}_{1}(w \cdot \mu-m p \beta): \hat{L}_{1}(\nu)\right]=\left[\hat{Z}_{1}(w \cdot \lambda-m p \beta): \hat{L}_{1}(\nu)\right]=0 \text { for } m \gg 0,
$$

so that (2) does in fact give a finite formula for the composition factors of $\operatorname{Im} \hat{\phi}$. Inducing still further from $G_{1} B$ to $G$ we obtain a $G$-homomorphism

$$
\phi: H^{l(w)}(w \cdot \lambda) \rightarrow H^{l(w)}(w \cdot \mu)
$$

and for $\lambda$ and $\mu$ generic one can argue as in [12, 3.10-3.12] to get the statement in Proposition 4.2 about the character of the image of $\phi$ :

$$
\operatorname{ch} \operatorname{Im} \phi=C_{\alpha}(\lambda)
$$

\section{REFERENCES}

1. H. H. Andersen, The strong linkage principle, J. Reine Angew. Math. 315 (1980), 53-59.

2. __ The first cohomology group of a line bundle on $G / B$, Invent. Math. 51 (1979), 287-296.

3.

4. Filtration of cohomology modules for Chevalley groups, Ann. Sci. Ecole Norm. Sup. 16 (1983), 495-528.

5. __ Extensions of modules for algebraic groups, Amer. J. Math. 106 (1984), 489-504.

6. E. Cline, B. Parshall and L. Scott, A Mackey imprimitivity theory for algebraic groups, Math. Z. 182 (1983), 447-471.

7. On injective modules for infinitesimal algebraic groups. I, J. London Math. Soc. (2) 31 (1985), 277-291.

8. S. Doty and J. Sullivan, On the structure of the higher cohomology modules of line bundles on $G / B$ (to appear).

9. J. E. Humphreys, On the structure of Weyl modules, Comm. Algebra 12 (1984), 2665-2677.

10. Cohomology of $G / B$ in characteristic $p$, Adv. in Math. (to appear).

11. J. C. Jantzen, Über das Dekompositionsverhalten gewisser modularer Darstellungen halbeinfacher Gruppen und ihrer Lie-Algebren, J. Algebra 49 (1977), 441-469.

12. __ Darstellungen halbeinfacher Gruppen und ihrer Frobenius-Kerne, J. Reine Angew. Math. 317 (1980), 157-199.

13. M. Koppinen, Homomorphisms between neighbouring Wevl modules (to appear).

School of Mathematics, Institute for Advanced Study, Princeton, New Jersey 08540

Permanent address: Matematisk Institut, Aarhus Universitet, DK-8000 Aarhus C, Denmark 